\title{
Using the Electronic Medical Record to Improve Education in Patients at Risk for Adrenal Insufficiency
}

\author{
Bahareh Schweiger, Philip Zeitler, Scott Eppley, Marguerite Swietlik, \\ and Jennifer Barker \\ The Children's Hospital, University of Colorado Denver, East 16th Avenue, B265 Aurora, CO 13123, USA \\ Correspondence should be addressed to Bahareh Schweiger, bahareh.schweiger@ucdenver.edu
}

Received 21 April 2010; Accepted 24 June 2010

Academic Editor: Jeffrey Gruen

Copyright $\odot 2010$ Bahareh Schweiger et al. This is an open access article distributed under the Creative Commons Attribution License, which permits unrestricted use, distribution, and reproduction in any medium, provided the original work is properly cited.

\begin{abstract}
Background. Adrenal insufficiency is a life-threatening event. It is recommended that patients with known adrenal insufficiency and their families receive careful and repeated education on sick-day glucocorticoid management. We hypothesized that the electronic medical record (EMR) can be used to improve patient education through automated provider notification. Methods. We established an automated electronic alert in the EMR that triggered in the outpatient endocrine clinic. The alert asked if stress dose education was reviewed at the visit. The response to this alert was evaluated between July 15, 2009 and February 19, 2010. Results. 128 unique patients had visits both prior to and following the implementation of the EMR alert. The alert was acknowledged in 58 unique patient visits. After the alert was implemented, 87/128 (68\%) of the patients had documentation in their record that stress dosing was reviewed. In the visit just prior to implementation of the alert, 48/128 (38\%) of the patient encounters showed written documentation of stress dose review. Conclusion. This report documents that an automated alert in the EMR can promote improved provider adherence to recommendations regarding ongoing education of patients for stress dosing of glucocorticoids. Whether this translates into better outcomes for patients remains to be seen.
\end{abstract}

\section{Introduction}

Primary and secondary adrenal insufficiency can be associated with life-threatening events during acute illness or underlying stress if prompt glucocorticoid replacement is not provided. However, despite formal patient education being included in routine endocrine care of these patients, adrenal crisis continues to occur in the pediatric population because of failure to increase glucocorticoid dosage appropriately [1]. In a retrospective review of 526 patients with adrenal insufficiency, $42 \%$ of patients had documentation of at least one crisis [2]. Common aliments such as gastrointestinal infection and fever [2] have been found to precipitate adrenal crisis, often leading to overnight hospitalization. In fact, $8 \%$ of diagnosed cases of Addison's disease will need hospital treatment for adrenal crisis annually [3]. In the pediatric population, inappropriate management of adrenal insufficiency during crisis is associated with excess mortality [4-7].
It is recommended that patients with known adrenal insufficiency and their families receive careful and repeated education on sick-day glucocorticoid management [8], including review of emergency injection of hydrocortisone [9]. They are also encouraged to purchase and wear an identification bracelet to ensure notification of the need for steroid replacement in case of incapacitation [10]. However, given the persistent occurrence of adrenal crisis due to inadequate management of stress dosing by families, traditional methods of stress dose education are clearly inadequate.

Review of the literature reveals that the combination of an automatic electronic provider alert, to bring awareness of patient specific issues, along with patient education is more effective than either alone in improving patient care. Coté et al. examined the impact of physician education, a computer alert, or both on the targeted use of GI prophylaxis for NSAID-related upper GI tract complications in highrisk patients discharged from hospital and found that the 


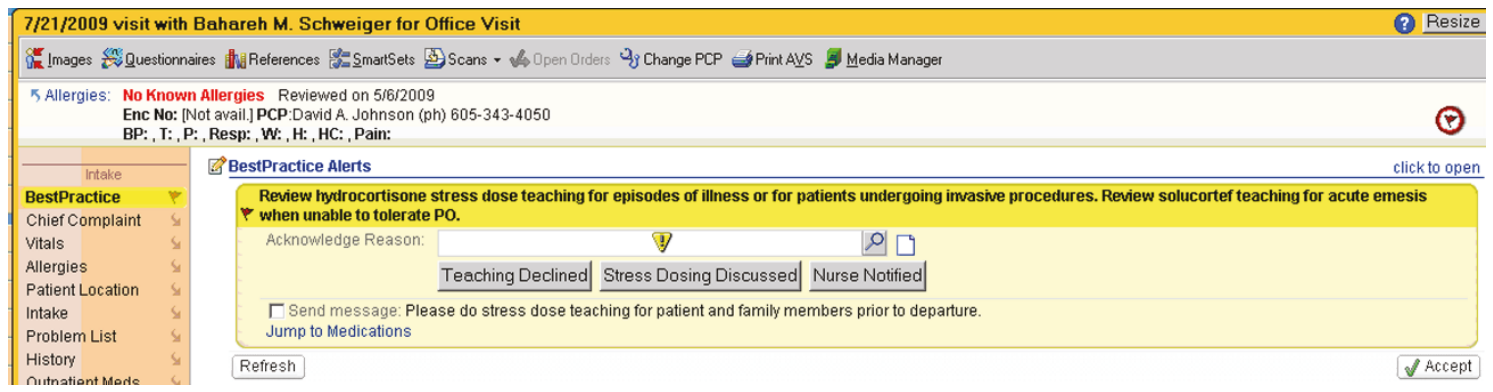

Figure 1: Best practice alert for pediatric endocrinology department. The alert triggers a reminder to review hydrocortisone stress dose teaching for episodes of illness or for patients undergoing invasive procedures.

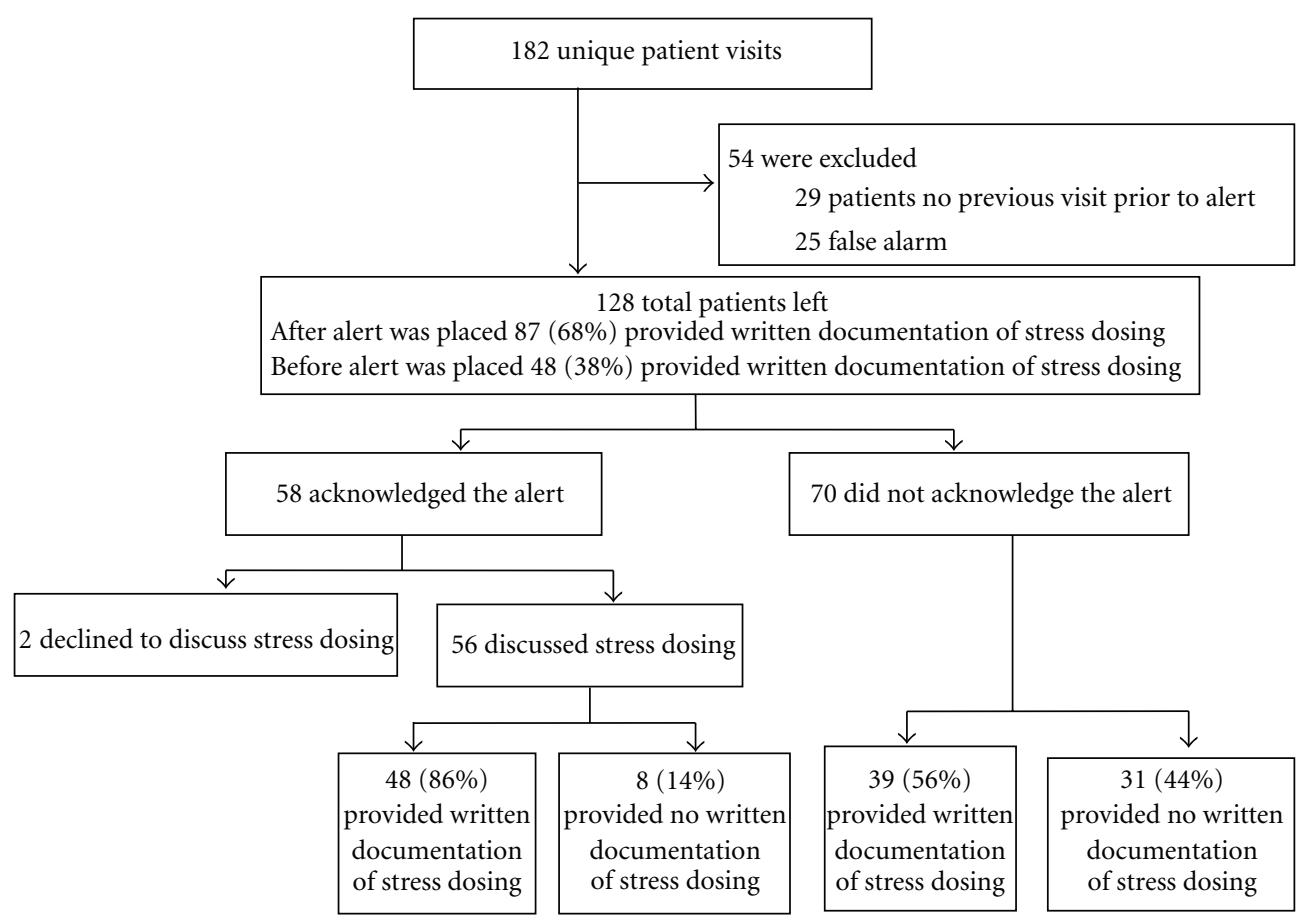

FIgURE 2: Flow chart of pediatric endocrinology stress dosing alert summary.

combination led to an increase in the use of gastroprotection [11]. Similarly, others have shown that automated alerts help decrease medication errors related to drug-laboratory interactions in an outpatient primary-care setting. Focusing on 18 high-volume and high-risk medications Steele et al. reported a significant increase in the percentage of time the provider stopped the ordering process and did not complete the medication order when an alert for an abnormal rule-associated laboratory result was displayed. The provider was also found to increase ordering of the rule-associated laboratory test when an alert was displayed [12]. On the other hand, others have found the use of EMR not as promising and report that patient follow-up remains unchanged despite automated notification of nonlife threatening abnormal laboratory results in the outpatient setting [13].

We decided to use our hospital EMR system to help improve the medical management of our patients with adrenal insufficiency and those on chronic steroid treatment. Our hospital EMR system is called Epic and it was instituted in 2007. It is a paperless system and essentially all patient charting within our institution are carried out using this system. We worked closely with our Clinical Application Services department to develop a Best Practice Alert. This report focuses on the design and consequences of the implementation of this alert.

\section{Methods}

We implemented an automated alert in the EMR on July 15, 2009. When the chart of a patient on steroids (oral or IV) is opened in the Endocrine Outpatient Clinic, an alert appears indicating that the patient is receiving glucocorticoid therapy and asking if stress dose education was reviewed at the visit (Figure 1). The alert then prompts the provider to respond 


\begin{tabular}{l}
\hline \multicolumn{2}{c}{ Primary Assessment } \\
BestPractice \\
Arrival Info \\
Triage Called \\
Triage Start \\
Chief Complaint \\
Allergy \\
History \\
Rapid Psych Flow... \\
Vitals Flowsheet \\
ED Notes \\
Triage Comnlete
\end{tabular}

BestPractice Alerts

This patient requires immediate Accucheck. Also consider stress dosing of hydrocortisone IMN. Call endocrine for

further recommendations.

Acknowledge Reason:

Therapy Started

$\Gamma$ Open order. Less than one year of age: Hydrocortisone sod succinate (conc: $50 \mathrm{mg} / \mathrm{mL}$ ) IVIM

$\Gamma$ Open order: One year thru 4 years of age: Hydrocortisone sod succinate (conc: $50 \mathrm{mg} / \mathrm{mL}$ ) IV/M.

(Last done by Nurse IP-Float Zztest at 10:03 AM on 2/23/2010)

$\Gamma$ Open order: 5 years of age and older. Hydrocortisone sod succinate (conc: $50 \mathrm{mg} / \mathrm{mL}$ ) $\mathrm{V} / \mathrm{M} \mathrm{M}$

(a)

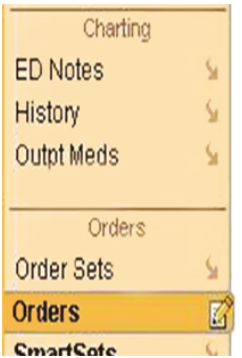

\section{ED Orders}

\begin{tabular}{|l|l|l|}
\hline Search for Orders: & Search & Pref List \\
\hline Medication
\end{tabular}

(b)

Figure 3: (a) Best practice alert for emergency department. An alert triggers that states the patient requires immediate Accucheck and to consider stress dosing of hydrocortisone IM/IV. It also suggests calling Endocrine for further recommendations. (b) When the age is selected, an order will open and choose the appropriate dose $(25 \mathrm{mg}, 50 \mathrm{mg}, 100 \mathrm{mg})$.

by clicking a button. An additional comment can be added. We asked that physicians then document in the visit note for that encounter whether stress dose education was reviewed. Study approval was obtained from the institutional review board at the University of Colorado Denver.

In addition, in order to improve communication throughout the hospital, we asked that proper hydrocortisone dosing for daily use, oral stress dose, and parenteral stress dose be listed at the end of every progress note.

We reviewed encounters from July 15, 2009 to February 19, 2010 and compared documentation of stress dosing teaching before and after implementation of the EMR alert.

\section{Results}

During this period, we identified 182 unique patient visits that activated the alert (Figure 2). Of these, 25 were erroneous activations for treatment with short courses of prednisone ( 2 weeks or less) for other medical conditions such as asthma or inflammatory bowel disease and so were excluded. Another 29 of the patients were excluded because they were initially seen in our clinic only after the alert was implemented. Of the 128 remaining patients who had visits both before and after implementation, the alert was acknowledged in 58 (45\%). From the 58 acknowledgments, there were 56 encounters in which the provider indicated that stress dosing was discussed with the patients and written documentation was provided in $48(86 \%)$ of these. The alert was not acknowledged in 70 encounters, in which 39 (56\%) showed written documentation of stress dose hydrocortisone education. Thus, after the alert was implemented, 87/128 $(68 \%)$ of the patients had documentation in their record that stress dosing was reviewed. In the visit just prior to initiation of the alert, written documentation of teaching was provided in 48/128 (38\%) of the corresponding patients. In addition, we found that the alert was acknowledged in $14 / 31(45 \%)$ encounters in the first month (July 15-August $15,2009)$ that it was implemented compared with the fifth month of implementation when it was acknowledged in 15/ 29 encounters (January 19-February 19, 2010).

\section{Discussion}

Our findings indicate that an EMR alert increased written documentation of physician review of stress dose education with families by more than 2-fold when the alert was acknowledged compared to encounters prior to the implementation of the alert. Of interest, documentation improved even for those encounters where the alert was not acknowledged, though to a lesser extent. It is possible that implementation of the alert alone may have increased general awareness of the problem. Though, the difference may also be merely due to chance. The reasons providers failed to acknowledge the alert are not known, but these results suggest that the alert is effective in improving review even when not directly acknowledged.

It should be noted that the lack of documentation prior to implementation of the alert is not necessarily evidence of lack of stress dosing review. It is possible that educations took place but were not documented. Also, the 
improved documentation of physician review presumably reflects actual education sessions, though we cannot exclude the possibility that providers documented review without performing the education. We can also not yet document improvement in management by families of episodes requiring stress dose glucocorticoid therapy. Further studies to track the frequency of ED visits and hospitalizations will be undertaken. In addition, even though it appears that there was no loss of efficacy of the alert over time we had too few events to adequately determine change over time and so this will also need to be further assessed in the future.

We have also received positive feedback from other departments regarding the format for written documentation regarding stress dosing. Traditional methods of "doubling" or "tripling" the hydrocortisone dosing during illness were ambiguous and fraught with error for both families and nonendocrine providers. By providing specific oral and parenteral stress doses prominently displayed in the medical record, care is facilitated and delay or underdosing is avoided. Improvements in care, however, have not yet been documented.

We have now established a similar alert within our hospital emergency department that will appear for any patient who presents with adrenal insufficiency and/or who is on steroids (oral or IV) (Figures 3(a) and 3(b)). The alert will trigger in triage and direct the nurse to obtain a finger stick blood glucose and alert the emergency physician immediately. The alert will also provide 3 weight-based stress doses for the physician. Evaluation of the success of this program in reducing time to treatment in the ED is underway. A similar initiative is being developed for the surgery department.

\section{Conclusion}

An automated alert in the EMR can promote improved provider adherence to recommendations regarding ongoing education of patients regarding stress dosing of glucocorticoids. Whether this translates into better outcomes for patients remains to be seen.

\section{References}

[1] D. I. Shulman, M. R. Palmert, and S. F. Kemp, "Adrenal insufficiency: still a cause of morbidity and death in childhood," Pediatrics, vol. 119, no. 2, pp. e484-e494, 2007.

[2] S. Hahner, M. Loeffler, B. Bleicken et al., "Epidemiology of adrenal crisis in chronic adrenal insufficiency: the need for new prevention strategies," European Journal of Endocrinology, vol. 162, no. 3, pp. 597-602, 2010.

[3] K. White and W. Arlt, "Adrenal crisis in treated Addison's disease: a predictable but under-managed event," European Journal of Endocrinology, vol. 162, no. 1, pp. 115-120, 2010.

[4] M. M. Erichsen, K. Løvås, K. J. Fougner et al., "Normal overall mortality rate in Addison's disease, but young patients are at risk of premature death," European Journal of Endocrinology, vol. 160, no. 2, pp. 233-237, 2009.

[5] J. W. Tomlinson, N. Holden, R. K. Hills et al., "Association between premature mortality and hypopituitarism," The Lancet, vol. 357, no. 9254, pp. 425-431, 2001.
[6] A. Waise and R. J. Young, "Pitfalls in the management of acute adrenocortical insufficiency: discussion paper," Journal of the Royal Society of Medicine, vol. 82, no. 12, pp. 741-742, 1989.

[7] K. Omori, K. Nomura, S. Shimizu, N. Omori, and K. Takano, "Risk factors for adrenal crisis in patients with adrenal insufficiency," Endocrine Journal, vol. 50, no. 6, pp. 745-752, 2003.

[8] S. Hahner and B. Allolio, "Management of adrenal insufficiency in different clinical settings," Expert Opinion on Pharmacotherapy, vol. 6, no. 14, pp. 2407-2417, 2005.

[9] A. H. Mulder, S. Nauta, G. F. Pieters, and A. R. M. M. Hermus, "Addisonian crisis in patients with known adrenal insufficiency: the importance of early intervention," Nederlands Tijdschrift voor Geneeskunde, vol. 152, no. 27, pp. 14971500, 2008.

[10] T. Kearney and C. Dang, "Diabetic and endocrine emergencies," Postgraduate Medical Journal, vol. 83, no. 976, pp. 79-86, 2007.

[11] G. A. Coté, J. P. Rice, W. Bulsiewicz et al., "Use of physician education and computer alert to improve targeted use of gastroprotection among NSAID users," American Journal of Gastroenterology, vol. 103, no. 5, pp. 1097-1103, 2008.

[12] A. W. Steele, S. Eisert, J. Witter et al., "The effect of automated alerts on provider ordering behavior in an outpatient setting," PLoS Medicine, vol. 2, no. 9, pp. 864-870, 2005.

[13] H. Singh, E. J. Thomas, D. F. Sittig et al., "Notification of abnormal lab test results in an electronic medical record: do any safety concerns remain?" The American Journal of Medicine, vol. 123, no. 3, pp. 238-244, 2010. 